\title{
Safety of cranial radiotherapy concurrent with tyrosine kinase inhibitors in non-small cell lung cancer patients: A systematic review
}

Citation for published version (APA):

Hendriks, L. E. L., Schoenmaekers, J., Zindler, J. D., Eekers, D. B. P., Hoeben, A., De Ruysscher, D. K. M., \& Dingemans, A-M. C. (2015). Safety of cranial radiotherapy concurrent with tyrosine kinase inhibitors in non-small cell lung cancer patients: A systematic review. Cancer Treatment Reviews, 41(7), 634-645. https://doi.org/10.1016/j.ctrv.2015.05.005

Document status and date:

Published: 01/07/2015

DOI:

10.1016/j.ctrv.2015.05.005

Document Version:

Publisher's PDF, also known as Version of record

Document license:

Taverne

Please check the document version of this publication:

- A submitted manuscript is the version of the article upon submission and before peer-review. There can be important differences between the submitted version and the official published version of record.

People interested in the research are advised to contact the author for the final version of the publication, or visit the DOI to the publisher's website.

- The final author version and the galley proof are versions of the publication after peer review.

- The final published version features the final layout of the paper including the volume, issue and page numbers.

Link to publication

\footnotetext{
General rights rights.

- You may freely distribute the URL identifying the publication in the public portal. please follow below link for the End User Agreement:

www.umlib.nl/taverne-license

Take down policy

If you believe that this document breaches copyright please contact us at:

repository@maastrichtuniversity.nl

providing details and we will investigate your claim.
}

Copyright and moral rights for the publications made accessible in the public portal are retained by the authors and/or other copyright owners and it is a condition of accessing publications that users recognise and abide by the legal requirements associated with these

- Users may download and print one copy of any publication from the public portal for the purpose of private study or research.

- You may not further distribute the material or use it for any profit-making activity or commercial gain

If the publication is distributed under the terms of Article $25 \mathrm{fa}$ of the Dutch Copyright Act, indicated by the "Taverne" license above, 


\title{
Safety of cranial radiotherapy concurrent with tyrosine kinase inhibitors in non-small cell lung cancer patients: A systematic review
}

\author{
Lizza E.L. Hendriks ${ }^{a}{ }^{*}$, Janna Schoenmaekers ${ }^{a}$, Jaap D. Zindler ${ }^{b}$, Danielle B.P. Eekers ${ }^{b}$, Ann Hoeben ${ }^{c}$, \\ Dirk K.M. De Ruysscher ${ }^{d}$, Anne-Marie C. Dingemans ${ }^{a}$
}

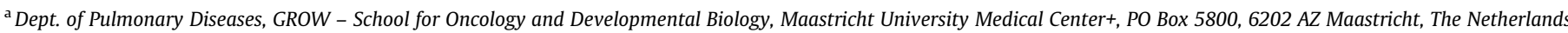

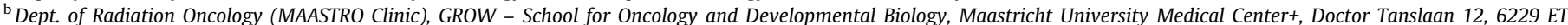
Maastricht, The Netherlands

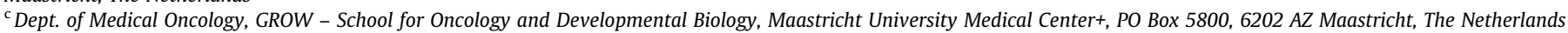

${ }^{\mathrm{d}}$ Dept. of Radiation Oncology, KU Leuven - University of Leuven, University Hospitals Leuven, Herestraat 49, 3000 Leuven, Belgium

\section{A R T I C L E I N F O}

\section{Article history:}

Received 1 April 2015

Received in revised form 5 May 2015

Accepted 6 May 2015

\section{Keywords:}

Tyrosine kinase inhibitor

NSCLC

Cranial radiotherapy

Neurotoxicity

EGFR

ALK

\begin{abstract}
A B S T R A C T
Recently, non-small cell lung cancer (NSCLC) has been partly subclassified into molecularly-defined oncogene "addicted" tumors for which targeted agents are available. Tyrosine kinase inhibitors (TKI) are currently approved for patients with an activating epidermal growth factor receptor (EGFR) mutation or anaplastic lymphoma kinase $(A L K)$ rearrangement. In these patients, brain metastases are often the first site of progression while on TKI treatment. The TKI may however still be active on extra-cranial sites and clinicians are thus faced with the question if the TKI may be continued during cranial radiotherapy. Advantages of combining TKI with cranial radiotherapy would be a possible synergistic effect on the brain metastases and the prevention of a systemic disease flare-up. A disadvantage is the possibly increased risk of (neuro)toxicity. The present systematic review addresses the toxicity of combining TKI with cranial radiotherapy in NSCLC patients.
\end{abstract}

(c) 2015 Elsevier Ltd. All rights reserved.

\section{Introduction}

Increasingly, new molecular features of non-small cell lung cancer (NSCLC) are being discovered, leading to an unprecedented growth of targeted agents. These are often tyrosine kinase inhibitors (TKI) [1]. Currently, TKI are approved for metastasized NSCLC patients with an activating epidermal growth factor receptor (EGFR) mutation or an anaplastic lymphoma kinase $(A L K)$ rearrangement, either as first line or beyond [2,3]. Examples are erlotinib, gefitinib, afatinib and icotinib (China only) for EGFR-mutations, and crizotinib and ceritinib (USA only) for $A L K$-rearrangements. Approximately $20-35 \%$ of these patients are diagnosed with brain metastasis at initial diagnosis and these patients are often amenable for initial treatment with TKI [4-8]. However, a substantial part will develop new brain metastasis or progression of brain metastasis during treatment. On erlotinib

\footnotetext{
* Corresponding author. Tel.: +31 (0)433871318; fax: +31 (0)433875051.

E-mail addresses: lizza.hendriks@mumc.nl (L.E.L. Hendriks), j.schoenmaekers@ mumc.nl (J. Schoenmaekers), jaap.zindler@maastro.nl (J.D. Zindler), danielle. eekers@maastro.nl (D.B.P. Eekers), ann.hoeben@mumc.nl (A. Hoeben), dirk.deruysscher@uzleuven.be (D.K.M. De Ruysscher), a.dingemans@mumc.nl (A.-M.C. Dingemans).
}

and gefitinib treatment $14-33 \%$ of patients develop (progression of) brain metastasis [9-15]. In patients with a survival beyond five years, this percentage increases to $52.9 \%$ [6]. On crizotinib treatment $70 \%$ of patients experience progression of brain metastases after an initial cerebral disease control rate of $60 \%$ (median time to intracranial progression: 7 months). $20 \%$ of patients without brain metastasis at initial NSCLC diagnosis develop brain metastasis during crizotinib treatment and this increases to about $58 \%$ in patients with a survival beyond three years $[6,8]$. In these patients, the brain is often the first and/or only site of progression (oligo-progression) $[8,12,14,16]$.

The TKI may however still be active on extra-cranial sites and clinicians are thus faced with the question if the TKI may be continued during cranial radiotherapy. Although there are pre-clinical studies suggesting that TKIs enhance radiation effects, the effects on normal tissues are unclear [17-20]. Data show that some molecular features of the tumor are not only related to response to TKI but also to radiation susceptibility of the tumor. As an example, tumors with activating EGFR-mutations not only show a high probability to respond to EGFR-TKI but also to radiation [21]. In current guidelines (ESMO 2014, NCCN 2014, ASTRO 2012) no recommendations are made regarding the concurrent use of TKI's and cranial radiotherapy in NSCLC patients with an activating 
mutation $[2,3,22,23]$. Frequently, TKI's are discontinued during cranial radiation because of (neuro)toxicity concerns. However, toxicity (e.g., radiation pneumonitis) does not seem to increase when EGFR-TKI are combined with thoracic radiotherapy in the majority of studies although some did report a higher incidence of grade 35 radiation pneumonitis [24-27]. Advantages of combining TKI with cranial radiotherapy would be a possible synergistic effect on the brain metastases and the prevention of a systemic disease flare-up. The latter has been described in both EGFR-mutated patients (23\% of patients, median time to disease flare-up 8 days, range 3-21 days) and in an $A L K$-translocated patient (time to disease flare-up 15 days) [28,29]. Among the factors associated with an increased risk for a disease flare-up was the presence of central nervous system (CNS) disease [28].

The aim of the present systematic review is to address the toxicity of combining TKI with cranial radiotherapy in NSCLC patients as, to the best of our knowledge, there is no systematic review on this topic. The focus will be on neurotoxicity. When possible, a daily practice advice will be formulated.

\section{Methods}

\section{Search strategy and selection criteria}

The literature search was performed following the PICO method [30] and is shown in Appendix 1. This search was used to identify studies in Pub Med, EMBASE, Web of Science and the Cochrane Library from 2001 until the search date in November 2014. Additionally, clinicaltrials.gov was searched to identify unpublished or ongoing clinical trials.

Selection criteria were established prior to the search and selection of articles. These included human only studies, including a minimum of 5 NSCLC patients treated with concurrent cranial radiotherapy and TKI's (EGFR: erlotinib, gefitinib, afatinib, icotinib, ALK: crizotinib, ceritinib and alectinib). As safety was the primary endpoint there was no restriction on the presence of a targetable mutation. Studies with whole brain radiotherapy (WBRT) as well as stereotactic radiosurgery/stereotactic radiotherapy (SRS/SRT) were included. Language was restricted to English, German and Dutch. Original articles and conference proceedings were included, reviews were excluded. Additionally, references of eligible articles were manually searched to find other relevant studies. All inclusion and exclusion criteria are summarized in Table 1.

\section{Outcomes}

One researcher (LH) conducted the search and selection of eligible studies. All articles were then evaluated by another independent reviewer (JS). When available, the following data were

Table 1

Inclusion criteria for this review.

$\begin{array}{ll}\text { Subjects included } & \text { Human only } \\ \text { Language } & \text { English, German, Dutch } \\ \text { Article type } & \text { Original article, conference proceeding } \\ \text { Number of patients } & \geqslant 5 \\ \text { Site of primary tumor } & \text { NSCLC } \\ \text { Tumor stage } & \text { IV } \\ \text { Treatment } & \text { WBRT and/or SRS/SRT concurrent with } \\ & \text { TKI (EGFR- or ALK-TKI) } \\ \text { Follow up period } & \text { All } \\ \text { Outcome } & \text { Safety/adverse events one of the outcomes measured }\end{array}$

Abbreviations: NSCLC: non-small cell lung cancer; WBRT: whole brain radiotherapy; SRS: stereotactic radiosurgery; SRT: stereotactic radiotherapy; TKI: tyrosine kinase inhibitor; EGFR: epidermal growth factor receptor; ALK: anaplastic lymphoma kinase. extracted from eligible studies by one researcher (LH) and independently by another researcher (JS): author, year of publication, original article or conference proceeding only, type of study, duration of study, number of included patients, EGFR mutation/ALK-translocation status available (yes/no) and results of mutation testing, dose cranial radiotherapy (WBRT and/or SRS/SRT), description of TKI used (including dosing and timing), safety and efficacy outcomes.

Data were extracted and tabulated independently (Appendix 2). Consensus was reached by discussion between reviewers when outcomes differed.

\section{Results}

\section{Search results}

The initial search in the four databases included 710 articles in total. Using Endnote and manual screening, 179 duplicate articles were excluded. Another 461 articles were excluded based on not relevant titles for this study, 70 articles were further screened. After reading of the abstracts, another 43 articles were excluded based on the exclusion criteria. Of the 27 remaining articles and conference proceedings, the whole article was read (not possible for conference proceeding). Based on the exclusion criteria, 11 articles and 3 conference proceedings were eligible to include in this review. With a manual search of the reference list of the included articles one other relevant article was found (flowchart in Fig. 1).

\section{Description and quality of the studies}

Of the 12 original articles and 3 conference proceedings that matched the selection criteria and were included in this review, 6 evaluated erlotinib concurrent with WBRT (one study combined WBRT with SRS) [31-36], 4 evaluated gefitinib concurrent with WBRT [37-40] and in 3 studies both drugs were studied [41-43]. In 2 studies icotinib concurrent with WBRT was studied [44,45]. For afatinib, crizotinib, ceritinib and alectinib no studies were found concurrent with cranial radiotherapy.

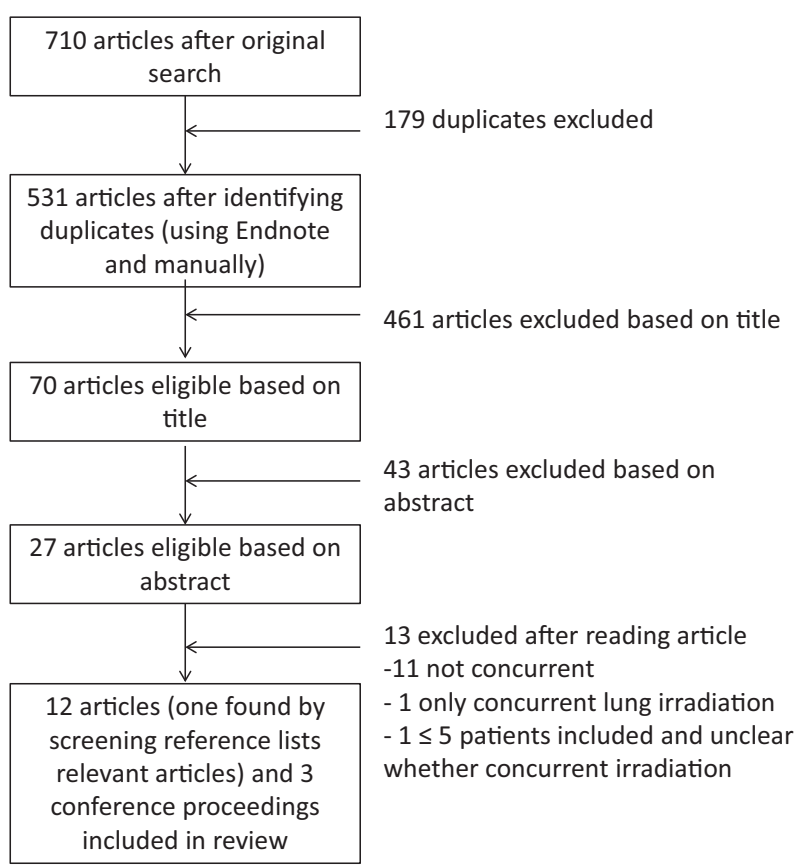

Fig. 1. Flowchart article selection. 
Five studies were retrospective [33,40-43]. 2 studies were phase I [32,45], 7 were phase II $[31,35-39,44]$, and there was only one phase III trial [34]. The phase III trial was a randomized, but not placebo controlled study [34]. 4 out of 7 phase II studies consisted of 2 arms $[31,36,38,39]$. In one of these studies patients were not randomized to one of the arms, but treatment allocation was based on whether EGFR-mutation status was determined. These patients (irrespective of EGFR-testing results) were allocated within the concurrent arm, the patients in which mutation analysis was not performed were allocated within the WBRT only arm [36]. Of the 4 phase II, 2 arm-studies only one study was a double blind, placebo controlled study [31].

There was one study in which only patients with an activating EGFR mutation were included [45] and there were 8 other studies in which at least part of the included patients were tested for activating EGFR mutations [31,35,36,39-41,43,44]. In these studies, percentage of EGFR-mutated patients (computed as number EGFR-mutated/total number of patients included) varied between $1.3 \%$ and $69.8 \%$.

For WBRT, the total radiation dose varied between 20 and $50 \mathrm{~Gy}$ in 4 (20 Gy) to 25 (50 Gy) fractions. EGFR-TKI were initiated from one week before the onset of cranial radiation to the first day of radiotherapy. In most studies, after WBRT, investigators could continue the EGFR-TKI at their own discretion [31-35,37-45]. Only in one study the EGFR-TKI was discontinued one month after completion of WBRT [36]. In the two-arm studies, the treatments that were compared varied between WBRT only (or combined with temozolomide or chemotherapy) and WBRT concurrent with EGFR-TKI, and EGFR-TKI only compared to WBRT concurrent with EGFR-TKI [31,34,36,38-41]. Primary outcomes ranged between overall survival (OS), (neurological) progression free survival ((n)PFS), local PFS (LPFS), intracranial response rate, toxicity and quality of life (QoL). All studies are summarized in Table 2. For the retrospective studies, all outcomes are listed as primary.

\section{Frequency and methods of toxicity evaluation}

In 3 out of 10 (30\%) prospective studies, toxicity was the primary objective $[32,39,45]$. In the study of Lind et al., neurotoxicity was not an end point of the trial; neurological examination was performed at baseline and was not specified during the follow-up although adverse events were recorded according to the Common Terminology Criteria of Adverse Events (CTCAE) criteria [32]. Wang et al. did not include specific neurocognitive functioning tests [39]. Zhou et al. (abstract only) only performed Mini Mental State Examinations (MMSE) up to 20 weeks after WBRT [45]. In the study of Lee et al., toxicity itself was not a primary objective, however nPFS was. This consisted of a clinical (MMSE, assessment of motor strength, visual acuity and gait) and radiological assessment. Adverse events according to the Common Terminology Criteria of Adverse Events (CTCAE) criteria were only recorded up to 28 days after finalizing the treatment [31].

From the other 6 prospective studies, Welsh et al. did the most extensive neurotoxicity evaluation. Neurological examination and MMSE were performed at baseline, at 6 months and at 12 months after treatment. Patients had also formal cognitive testing (neurological examination, Hopkins Verbal Learning Test-Revised, Trail Making Test Part A and B, Multilingual Aphasia Examination Controlled Oral Word Association) before enrollment, within 14 days of WBRT completion and at each follow-up visit (at 1 month and then every 3 months) [35]. In the study of Pesce et al., cognitive function (MMSE, Trail Making Test part B, EORTC QLQ-C30 cognitive function subscale) was assessed prior to start and on day one of cycles 2, 3 and 5 [38]. In the study of Ma et al., neurological examination was performed weekly during concurrent treatment [37]. In the other studies, neurotoxicity was not a specific item and toxicity was scored according to the CTCAE criteria $[34,36,37,44]$. Methods and frequency of toxicity assessment are summarized in Table 3. Only prospective studies are summarized, as in the retrospective studies there was no specified protocol for follow-up.

\section{Toxicity outcomes}

All outcomes are summarized in Table 4.9 trials ( 3 retrospective) specifically mentioned neurotoxicity $[32-35,37,40,41,45]$ of which 5 reported that there was no increased neurotoxicity of the concurrent treatment $[32,35,40,41,45]$. However, in one of these studies 2 questionable late neurotoxicity events were reported: one 74-year old male developed dementia two years after study completion and one 56-year old female had 5 months after study completion intracranial progression for which she received SRS, she developed brain necrosis thereafter. Other contributing factors for these events were older age (possibly unrecognized cognitive impairment) in the first patient and the use of SRS in the second [35]. In one study 5\% grade 3 dizziness for both the WBRT only and the WBRT + erlotinib group was reported but no late neurotoxicity. Duration of dizziness was not mentioned [36]. In one retrospective study $(N=8) 3$ patients had mental status change. Two of these patients also had grade 3 hyponatriemia and an intercurrent infection as a possible contributing factor for the mental status change [33]. In a prospective randomized study of WBRT together with SRS and combined with erlotinib, temozolomide or no systemic treatment, grade 4 brain necrosis and grade 5 hemorrhagic stroke both occurred in $1 / 41$ patients in the erlotinib arm. Grade 3 confusion and ataxia were also found, but number of patients was not specifically mentioned [34]. In a single arm prospective study $(N=21) 14 \%$ grade 3 headache was reported, but grade $4-5$ toxicities did not occur [37]. In the other 6 studies neurotoxicities were not specifically described. However, all grade/grade 3-5 toxicities were mentioned and these did not include grade 3-5 neurotoxicities. Time to resolution of toxicities was not mentioned [31,38,39,42-44].

\section{Discussion}

A relatively high percentage of EGFR-mutated and $A L K$-rearranged patients will develop brain metastases during the course of their disease, often while on TKI treatment $[9-15,46]$. A possible explanation for this high percentage is that the first generation TKI's do not achieve therapeutic concentrations in the brain due to (relative) inability to cross the blood-brain barrier [47-49]. In this situation, extra cranially located cancer cells are often still dependent on EGFR- or ALK-signaling and are responding to TKI's [12]. In patients with brain metastases both WBRT and SRS/SRT can be considered, mainly dependent on the number/volume of brain metastases and the performance status of the patient [3]. In current guidelines, no advice regarding TKI use during cranial radiotherapy is given $[2,3,22,23]$. To our knowledge, the present study was the first to systematically review the literature in order to evaluate the safety of concurrent cranial radiotherapy and TKI. For ALK-TKI, no studies were found. For EGFR-TKI, 15 studies were found of which 5 were retrospective [31-45]. In only 9 studies, presence of an activating EGFR-mutation was evaluated with varying percentages $(1.3-100 \%)[31,35,36,39-41,43-45]$. Only one study was a phase III study [34] and only 3 out of 7 phase II studies were randomized 2 arm studies [31,38,39]. In the identified papers, treatments studied varied between WBRT only, WBRT concurrent with EGFR-TKI (in one study also combined with SRS) and EGFRTKI only. No studies were found for SRS without WBRT concurrently with TKI. No studies were identified in which patients with 
Table 2

Trial characteristics of the included studies.

\begin{tabular}{|c|c|c|c|c|c|c|c|}
\hline Trial, year & Trial type & N & $\begin{array}{l}\text { EGFR mutation } \\
\text { analysis }\end{array}$ & WBRT/SRS treatment & TKI treatment ( \pm comparator) & $\begin{array}{l}\text { Primary study } \\
\text { objective }\end{array}$ & Secondary study objectives \\
\hline \multicolumn{8}{|c|}{ Erlotinib and $W B R T+S R S$} \\
\hline $\begin{array}{l}\text { Sperduto et al. } \\
\text { (2013) [34] }\end{array}$ & $\begin{array}{l}\text { Phase III, multicenter, } 3 \\
\text { arms, randomized, not } \\
\text { placebo controlled, } \\
\text { 2004-2009 }\end{array}$ & 126 (planned 381) & Not tested & $\begin{array}{l}\text { WBRT } 35 \text { Gy (15f/ } \\
2.5 \text { Gy) combined with } \\
\text { SRS to brain mets: } 18- \\
24 \text { Gy }\end{array}$ & $\begin{array}{l}\text { Arm A: WBRT/SRS } \\
\text { Arm B: WBRT/SRS + temozolomide } 75 \mathrm{mg} / \\
\mathrm{m}^{2} / \text { day } \\
\text { Arm C: WBRT/SRS + erlotinib } 150 \mathrm{mg} / \text { day, } \\
\text { start day } 1 \text { of RT, continuation after WBRT } \\
\text { on discretion of investigator }\end{array}$ & OS & $\begin{array}{l}\text { PFS intracranial } \\
\text { PS at } 6 \text { months } \\
\text { Steroid dependence } \\
\text { Cause of death } \\
\text { Toxicity mentioned in } \\
\text { results, not in objectives }\end{array}$ \\
\hline \multicolumn{8}{|l|}{ Erlotinib and WBRT } \\
\hline $\begin{array}{l}\text { Lind et al. } 2009 \\
\text { [32] }\end{array}$ & $\begin{array}{l}\text { Phase I, single arm, } \\
2006-2007\end{array}$ & 11 & Not tested & 30 Gy (10f/3 Gy) & $\begin{array}{l}4 \text { patients: erlotinib } 100 \mathrm{mg} / \mathrm{d}, 7 \text { patients } \\
150 \mathrm{mg} / \text { day, started one week before, } \\
\text { concurrent with WBRT, then maintenance } \\
150 \mathrm{mg} / \text { day }\end{array}$ & Toxicity (CTCAE v 3.0) & $\begin{array}{l}\text { PFS (intra- and extracranial) } \\
\text { OS }\end{array}$ \\
\hline $\begin{array}{l}\text { Olmez et al. } \\
\text { (2010)[33] }\end{array}$ & $\begin{array}{l}\text { Retrospective, single } \\
\text { center, 2007-2009 }\end{array}$ & 8 & Not tested & $\begin{array}{l}35(15 f / 2.5 \mathrm{~Gy})-40 \mathrm{~Gy} \\
(20 \mathrm{f} / 2 \mathrm{~Gy})\end{array}$ & $\begin{array}{l}\text { Erlotinib } 150 \mathrm{mg} / \text { day, started within } \\
5 \text { days of RT }\end{array}$ & $\begin{array}{l}\text { Outcome (response } \\
\text { rate intra- and } \\
\text { extracranial) } \\
\text { Toxicity (CTCAE v 3.0) }\end{array}$ & None \\
\hline $\begin{array}{l}\text { Welsh et al. (2013) } \\
\text { [35] }\end{array}$ & $\begin{array}{l}\text { Phase II, } 2 \text { centers, } \\
\text { single arm, 2006-2010 }\end{array}$ & 40 & $\begin{array}{l}17 / 40 \text { tested } \\
9 / 17 \text { EGFR+ }\end{array}$ & $\begin{array}{l}\text { First } 10 \text { patients } 30 \mathrm{~Gy} \\
(10 \mathrm{f} / 3 \mathrm{~Gy}) \text {, others } \\
35 \mathrm{~Gy}(14 \mathrm{f} / 2.5 \mathrm{~Gy})\end{array}$ & $\begin{array}{l}\text { Erlotinib } 150 \mathrm{mg} / \mathrm{day} \text {, started one week } \\
\text { before, concurrent with WBRT, then } \\
\text { maintenance } 150 \mathrm{mg} / \text { day }\end{array}$ & OS & $\begin{array}{l}\text { Radiologic response } \\
\text { Toxicity (CTCAE v } 3.0 \text { AND } \\
\text { neurological evaluation) }\end{array}$ \\
\hline $\begin{array}{l}\text { Zhuang et al. } \\
\text { (2013) [36] }\end{array}$ & $\begin{array}{l}\text { Phase II, single center, } \\
2 \text { arms, not } \\
\text { randomized, not } \\
\text { placebo controlled, } \\
\text { 2009-2011 }\end{array}$ & 54 & $\begin{array}{l}23 / 54 \text { tested } \\
11 / 23 E G F R^{+}\end{array}$ & $30 \mathrm{~Gy}(10 \mathrm{of} / 3 \mathrm{~Gy})$ & $\begin{array}{l}\text { Arm A: EGFR mutation status tested (not } \\
\text { necessarily activation mutation) erlotinib } \\
150 \mathrm{mg} / \text { day from first day of WBRT to } \\
\text { month after end of WBRT } \\
\text { Arm B: only WBRT }\end{array}$ & $\begin{array}{l}\text { Intracranial response } \\
\text { rate } \\
\text { LPFS }\end{array}$ & $\begin{array}{l}\text { Toxicity (CTCAE v } \\
\text { unknown) } \\
\text { PFS } \\
\text { OS }\end{array}$ \\
\hline $\begin{array}{l}\text { Lee et al. (2014) } \\
\text { [31] }\end{array}$ & $\begin{array}{l}\text { Phase II, multicenter, } \\
\text { two-stage, randomized, } \\
\text { double blind, placebo } \\
\text { controlled, 2009-2010 }\end{array}$ & 80 & $\begin{array}{l}35 / 80 \text { tested } \\
1 / 35 E G F R+\end{array}$ & 20 Gy (5f/4 Gy) & $\begin{array}{l}\text { Arm A: erlotinib } 100 \mathrm{mg} / \text { day started } 1 \mathrm{st} \\
\text { day of WBRT, after completing WBRT } \\
\text { increased to } 150 \mathrm{mg} / \text { day maintenance } \\
\text { Arm B: placebo starting on } 1 \text { st day of } \\
\text { WBRT }\end{array}$ & 2-Month nPFS & $\begin{array}{l}\text { Toxicity (CTCAE v } 3.0 \text { ) } \\
\text { QoL }\end{array}$ \\
\hline \multicolumn{8}{|l|}{ Gefitinib and WBRT } \\
\hline $\begin{array}{l}\text { Zeng et al. (2012) } \\
\text { [40] }\end{array}$ & $\begin{array}{l}\text { Retrospective, single } \\
\text { center, 2005-2009 }\end{array}$ & 90 & $\begin{array}{l}20 / 90 \text { tested } \\
12 / 20 E G F R+\end{array}$ & 40 Gy (20f/2 Gy) & $\begin{array}{l}\text { Arm A Gefitinib } 250 \mathrm{mg} / \text { day with } \\
\text { concurrent WBRT, afterwards } 250 \mathrm{mg} / \text { day } \\
\text { continued } \\
\text { Arm B: gefitinib } 250 \mathrm{mg} / \text { day }\end{array}$ & $\begin{array}{l}\text { "Efficacy and toxicity of } \\
\text { gefitinib alone } \\
\text { compared to } \\
\text { concurrent with } \\
\text { WBRT" }\end{array}$ & \\
\hline $\begin{array}{l}\text { Pesce et al. (2012) } \\
\text { [38] }\end{array}$ & $\begin{array}{l}\text { Open label, } \\
\text { randomized, } 2 \text { stage, } \\
\text { Phase II, multicenter, } \\
\text { 2005-2009 }\end{array}$ & 59 & Not tested & $30 \mathrm{~Gy}(10 \mathrm{f} / 3 \mathrm{~Gy})$ & $\begin{array}{l}\text { Arm A: gefitinib } 250 \mathrm{mg} / \text { day concurrent } \\
\text { with WBRT, afterwards } 250 \mathrm{mg} / \mathrm{day} \\
\text { continued } \\
\text { Arm B: temozolomide } 75 \mathrm{mg} / \mathrm{m} 2 \\
\text { concurrent with WBRT, afterwards } 75 \mathrm{mg} / \\
\mathrm{m} 2 \text { continued }\end{array}$ & OS & $\begin{array}{l}\text { PFS (intra- and extracranial) } \\
\text { QoL (including cognitive } \\
\text { function, toxicity, } \\
\text { tolerability) }\end{array}$ \\
\hline $\begin{array}{l}\text { Wang et al. (2014) } \\
\text { [39] }\end{array}$ & $\begin{array}{l}\text { Prospective, } \\
\text { randomized, phase II, } \\
\text { number of centers } \\
\text { unknown, no placebo, } \\
2010-2013\end{array}$ & 73 & $\begin{array}{l}\text { Number tested } \\
\text { unknown, in gefitinib } \\
\text { group } 9 / 37 E G F R^{+}\end{array}$ & $50 \mathrm{~Gy}(25 \mathrm{f} / 2 \mathrm{~Gy})$ & $\begin{array}{l}\text { Arm A: gefitinib } 250 \mathrm{mg} / \text { day start first day } \\
\text { of WBRT } \\
\text { Arm B: VM- } 26100 \mathrm{mg} / \text { day iv dag } 1-3 \text {, } \\
\text { cisplatin } 25 \mathrm{mg} / \mathrm{m} 2 \text { iv dag } 1-3,2 \text { cycles, } \\
\text { every cycle } 21 \text { days. Start first day of } \\
\text { WBRT }\end{array}$ & $\begin{array}{l}\text { Intracranial response } \\
\text { rate } \\
\text { OS } \\
\text { Toxicity (CTCAE v 3.0) }\end{array}$ & \\
\hline $\begin{array}{l}\text { Ma et al. (2009) } \\
\text { [37] }\end{array}$ & $\begin{array}{l}\text { Phase II, single arm, } \\
\text { single center, 2005- } \\
2007\end{array}$ & 21 & Not tested & $40 \mathrm{~Gy}(20 \mathrm{f} / 2 \mathrm{~Gy})$ & $\begin{array}{l}\text { Gefitinib } 250 \mathrm{mg} / \text { day concurrent with } \\
\text { WBRT, afterwards } 250 \mathrm{mg} / \text { day continued }\end{array}$ & $\begin{array}{l}\text { Intracranial response } \\
\text { rate } \\
\text { QoL }\end{array}$ & $\begin{array}{l}\text { Toxicity (CTCAE) } \\
\text { PFS } \\
\text { OS }\end{array}$ \\
\hline
\end{tabular}




\begin{tabular}{|c|c|c|c|c|c|c|c|}
\hline Trial, year & Trial type & $N$ & $\begin{array}{l}\text { EGFR mutation } \\
\text { analysis }\end{array}$ & WBRT/SRS treatment & TKI treatment ( \pm comparator) & $\begin{array}{l}\text { Primary study } \\
\text { objective }\end{array}$ & Secondary study objectives \\
\hline \multicolumn{8}{|c|}{ Erlotinib or gefitinib and WBRT } \\
\hline $\begin{array}{l}\text { Cai et al. (2013) } \\
\text { [41] }\end{array}$ & $\begin{array}{l}\text { Retrospective, single } \\
\text { center, 2009-2012 }\end{array}$ & 157 & All tested, 43 EGFR+ & $\begin{array}{l}30-42 \text { Gy }(10-14 f / \\
3 \text { Gy) }\end{array}$ & $\begin{array}{l}\text { Arm A: erlotinib } 150 \mathrm{mg} / \text { day or gefinitib } \\
250 \mathrm{mg} / \text { day concurrent with WBRT. } \\
\text { Startdate TKI not mentioned } \\
\text { Arm B: only WBRT }\end{array}$ & $\begin{array}{l}\text { Intracranial response } \\
\text { rate } \\
\text { PFS } \\
\text { OS } \\
\text { Toxicity (CTCAE and } \\
\text { RTOG) }\end{array}$ & \\
\hline $\begin{array}{l}\text { Lee et al. (2012) } \\
\text { [43] }\end{array}$ & $\begin{array}{l}\text { Retrospective, } 2 \\
\text { centers, 2003-2011 }\end{array}$ & 43 & All tested, 30 EGFR+ & $\begin{array}{l}30(10 \mathrm{f} / 3 \mathrm{~Gy})-40 \mathrm{~Gy} \\
(20 \mathrm{f} / 2 \mathrm{~Gy}), \text { with/ } \\
\text { without boost } 50- \\
60 \mathrm{~Gy} \text { on metastases }\end{array}$ & $\begin{array}{l}44 \% \text { EGFR-TKI concurrent with WBRT, in } \\
\text { EGFR + group } 50 \% \text {, dose erlotinib or } \\
\text { gefitinib not mentioned }\end{array}$ & $\begin{array}{l}\text { "impact of EGFR } \\
\text { mutations on RT" } \\
\text { (intracranial response } \\
\text { rate, toxicity (CTCAE v } \\
\text { 3.0), OS) }\end{array}$ & $\begin{array}{l}\text { Effect of concurrent chemo } \\
\text { or EGFR-TKI }\end{array}$ \\
\hline $\begin{array}{l}\text { Inamasu (conf } \\
\text { abstract) } \\
\quad(2012)[42]\end{array}$ & $\begin{array}{l}\text { Retrospective, single } \\
\text { center, 2005-2011 }\end{array}$ & 18 & Not mentioned & Not mentioned & $\begin{array}{l}\text { Erlotinib or gefitinib concurrent with } \\
\text { WBRT, dose not mentioned }\end{array}$ & Toxicity & \\
\hline \multicolumn{8}{|l|}{ Icotinib and WBRT } \\
\hline $\begin{array}{l}\text { Zhou et al. (conf } \\
\text { abstract) } \\
(2014)[45]\end{array}$ & $\begin{array}{l}\text { Phase I, single center, } \\
\text { open label, dose } \\
\text { finding, 2011-2013 }\end{array}$ & 15 & All tested, all EGFR+ & 37.5 Gy (15f/2.5 Gy) & $\begin{array}{l}3+3 \text { design, icotinib } 125,250,375 \text {, } \\
500 \text { mg TID. Start } 7 \text { days before WBRT, } \\
\text { concurrent with WBRT, continuation after } \\
\text { WBRT }\end{array}$ & $\begin{array}{l}\text { Toxicity } \\
\text { Neurocognitive } \\
\text { functioning (MMSE) } \\
\text { within } 20 \text { weeks of } \\
\text { WBRT }\end{array}$ & ORR, DCR, PFS \\
\hline $\begin{array}{l}\text { Yun et al. (conf } \\
\text { abstract) } \\
\text { (2013) [44] }\end{array}$ & $\begin{array}{l}\text { Phase II, single arm, } \\
\text { open label, 2012-2013 }\end{array}$ & 20 & $\begin{array}{l}\text { All tested, number of } \\
E G F R+\text { not mentioned }\end{array}$ & 30 Gy (10f/3 Gy) & $\begin{array}{l}\text { Icotinib } 125 \mathrm{mg} \text { TID concurrent with } \\
\text { WBRT }\end{array}$ & $\begin{array}{l}\text { Response rate } \\
\text { intracranial }\end{array}$ & $\begin{array}{l}\text { PFS, OS, QoL } \\
\text { Toxicity } \\
\text { Relationship response and } \\
\text { EGFR-mutation } \\
\text { CSF concentration of } \\
\text { icotinib }\end{array}$ \\
\hline
\end{tabular}

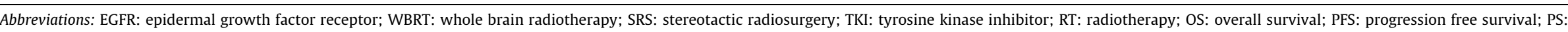

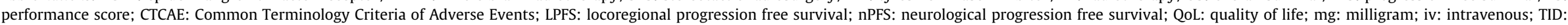
three times a day; MMSE: mini mental state examination; ORR: overall response rate; DCR: disease control rate; CSF: cerebrospinal fluid. 
Table 3

Methods of toxicity evaluation in the included studies.

\begin{tabular}{|c|c|c|c|c|}
\hline Trial, year & $\begin{array}{l}\text { Toxicity } \\
\text { primary } \\
\text { endpoint }\end{array}$ & Frequency of toxicity assessment & $\begin{array}{l}\text { Method of general } \\
\text { toxicity assessment }\end{array}$ & $\begin{array}{l}\text { Method of neurocognitive toxicity } \\
\text { assessment }\end{array}$ \\
\hline $\begin{array}{l}\text { Erlotinib and WBRT }+S R S \\
\text { Sperduto et al. (2013) [34] }\end{array}$ & No & Monthly during protocol therapy & CTCAE v 3.0 & Not specifically assessed \\
\hline $\begin{array}{l}\text { Erlotinib and WBRT } \\
\text { Lind et al. (2009) [32] }\end{array}$ & Yes & $\begin{array}{l}\text { Week } 1 \text { and } 2 \text { of WBRT } \\
\text { Then at } 2 \text { weeks, } 4 \text { weeks, } 2 \text { months } \\
\text { Then every } 2 \text { months until disease } \\
\text { progression and/or death }\end{array}$ & CTCAE v 3.0 & $\begin{array}{l}\text { Baseline assessment: neurological } \\
\text { examination } \\
\text { Otherwise only CTCAE }\end{array}$ \\
\hline $\begin{array}{l}\text { Olmez et al. (2010) [33] } \\
\text { Welsh et al. (2013) [35] }\end{array}$ & No & $\begin{array}{l}\text { Retrospective study, not specified } \\
\text { Baseline, within } 14 \text { days of WBRT } \\
\text { completion } \\
\text { Afterwards at } 1 \text { month, then every } \\
3 \text { months }\end{array}$ & CTCAE v 3.0 & $\begin{array}{l}\text { Neurological examination, MMSE (at } \\
0,6 \text { and } 12 \text { months) } \\
\text { Formal cognitive testing (thorough } \\
\text { neurological examination, Hopkins } \\
\text { Verbal Learning Test-Revised, Trail } \\
\text { Making Test Part A and B, } \\
\text { Multilingual Aphasia Examination } \\
\text { Controlled Oral Word Association) }\end{array}$ \\
\hline Zhuang et al. (2013) [36] & No & $\begin{array}{l}\text { Baseline, } 1 \text { month after WBRT } \\
\text { Afterwards every } 2-3 \text { months }\end{array}$ & CTCAE v 3.0 & None \\
\hline Lee et al. (2014) [31] & No & $\begin{array}{l}\text { CTCAE evaluation only the first } 28 \text { days. } \\
\text { Neurological exam: before randomisation } \\
\text { first } 8 \text { weeks } 2 \text {-weekly } \\
\text { Then monthly the first } 12 \text { months, } \\
\text { afterwards 2-montly }\end{array}$ & CTCAE v 3.0 & $\begin{array}{l}\text { MMSE } \\
\text { Clinical assessment of motor } \\
\text { strength, visual acuity and gait }\end{array}$ \\
\hline $\begin{array}{l}\text { Gefitinib and WBRT } \\
\text { Zeng et al. (2012) [40] }\end{array}$ & & Retrospective study, not specified & & \\
\hline Pesce et al. (2012) [38] & No & $\begin{array}{l}\text { Baseline } \\
\text { Day } 1 \text { of cycle } 2,3 \text { and } 5\end{array}$ & CTCAE v 3.0 & $\begin{array}{l}\text { MMSE, Trail Making Test part B and } \\
\text { EORTC QLQ-C30 cognitive function } \\
\text { subscale }\end{array}$ \\
\hline Wang et al. (2014) [39] & Yes & $\begin{array}{l}\text { Weekly during concurrent treatment } \\
\text { Then 3-montly interval for the first year } \\
\text { Afterwards every 3-6 months }\end{array}$ & CTCAE v 3.0 & None \\
\hline Ma et al. (2009) [37] & No & $\begin{array}{l}\text { Weekly during concurrent treatment } \\
\text { Afterwards not very well specified, } \\
\text { presumably every month }\end{array}$ & $\begin{array}{l}\text { CTCAE, version not } \\
\text { mentioned }\end{array}$ & Neurological examination \\
\hline $\begin{array}{l}\text { Erlotinib or gefitinib and WBRT } \\
\text { Cai et al. (2013) [41] } \\
\text { Lee et al. (2012) [43] } \\
\text { Inamasu (conf abstract) (2012) [42] }\end{array}$ & & $\begin{array}{l}\text { Retrospective study, not specified } \\
\text { Retrospective study, not specified } \\
\text { Retrospective study, not specified }\end{array}$ & & \\
\hline $\begin{array}{l}\text { Icotinib and WBRT } \\
\text { Zhou et al. (conf abstract) (2014) [45] }\end{array}$ & Yes & Not mentioned in abstract & $\begin{array}{l}\text { CTCAE, version not } \\
\text { mentioned }\end{array}$ & MMSE up to 20 weeks after WBRT \\
\hline Yun et al. (conf abstract) (2013) [44] & No & Not mentioned in abstract & $\begin{array}{l}\text { Not mentioned in } \\
\text { abstract }\end{array}$ & Not mentioned in abstract \\
\hline
\end{tabular}

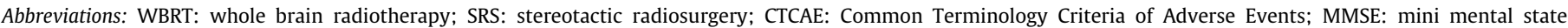
examination.

an activating mutation/translocation developed brain metastases while on TKI treatment and were subsequently randomized to cranial radiotherapy with concurrent TKI or cranial radiotherapy with temporarily discontinuation of the TKI. Primary outcomes varied and outcome data were measured and reported in a non-uniform way. Based on these data, WBRT concurrent with EGFR-TKI does not seem to increase neurotoxicity, although no firm conclusions can be made. However, one should be cautious in interpreting the neurological toxicity data from most of these studies, as in only two studies extensive neurocognitive tests were performed $[35,38]$. In both of these studies neurotoxicity was not the primary endpoint; one study was a single arm study and the other was not powered to detect neurotoxicity differences. Moreover, in the two arm study there was no comparator arm without an active systemic drug (one arm gefitinib concurrently with WBRT, the other temozolomide concurrently with WBRT) [38]. As such, subtle changes in neurocognitive functioning due to concurrent TKI and cranial radiotherapy cannot be excluded. Furthermore, WBRT combined with SRS, concurrent with EGFR-TKI does seem to increase neurotoxicity when compared to historical data for WBRT and
SRS without concurrent systemic treatment. In the study arm with concurrent EGFR-TKI, grade 3 confusion and ataxia (percentage not mentioned), grade 4 brain necrosis (2.4\%) and grade 5 stroke (2.4\%) were reported [34]. In a previous study without systemic treatment, acute toxicities were grade 3 in $2 \%$ of patients, grade 4 in $1 \%$ and none had grade 5 toxicities [50]. Non-neurological toxicities seem to increase with the concurrent use of EGFR-TKI when compared to monotherapy EGFR-TKI or WBRT. EGFR-TKI's are known to cause grade $\geqslant 3$ toxicities, like rash (3-13\%), diarrhea $(0-25 \%)$, fatigue $(0-19 \%)$, nausea (3-6\%), vomiting (3-5\%) and interstitial lung disease (1.2\%, all grades) [51-53]. When compared to the $3-13 \%$ grade $\geqslant 3$ rash encountered with monotherapy EGFRTKI, this percentage is slightly higher in the concurrent treated patients in this review (9-20\%) [31-35,43]. Grade $\geqslant 3$ rash was more often encountered with erlotinib than with gefitinib as is also known from literature, possibly because erlotinib is given at the maximum tolerated dose (MTD) and gefitinib is given at approximately one third of the MTD [54]. Although location of rash was not described, it is possible that this occurred on the scalp due to the concurrent WBRT. The same holds true for grade $\geqslant 3$ mucositis, 
Trial results of the included studies.

$\begin{array}{lll}\text { Arms specified when } & \text { Neurological safety outcome } & \text { All other grade 3-5 toxicities }\end{array}$

Efficacy outcome

\begin{tabular}{l}
\hline Trial, year \\
\hline Erlotinib and WBRT + SRS \\
Sperduto et al. \\
\hline
\end{tabular}

Retrospective

$N$

necessary

Neurological safety outcome

All other grade $3-5$ toxicities

\section{A: WBRT/SRS}

B: WBRT/

SRS + temozolomide

C: WBRT/SRS + erlotinib

Erlotinib and WBRT

[32]

Olmez et al. (2010)

[33]

Welsh et al. (2013)

[35]

Single arm

Single arm

Single arm

Zhuang et al.

(2013) [36

Lee et al. (2014)

[31]

Gefitinib and WBRT

Zeng et al. (2012)

[40]

Pesce et al. (2012)

[38]

Wang et al. (2014)

[39]

$\begin{array}{ll}\text { 126 (planned } & \text { A: WBRT/SRS } \\ \text { 381) } & \text { B: WBRT/ } \\ & \text { SRS + temozolomide } \\ & \text { C: WBRT/SRS + erlotinib }\end{array}$

WBRT + gefitini

$B$ : gefitinib only

A: WBRT + gefitinib

B: WBRT + temozolomide

A: WBRT + gefitinib
B: WBRT + VMP
Arm C: grade 3 confusion and ataxia (\% no . grade 5 stroke (2.4\%)

No treatment related neurotoxicity

Mental status change (37.5\%)

No direct neurotoxicity (measurements including neurocognitive testing)

$2.5 \%$ gr 3 headache

2 patients questionable late neurotoxicity

( 1 male, aged 74 developed dementia

2 years after study completion, one

patient developed radiation necrosis after

intracranial PD for which she received

SRS)

A: WBRT + erlotinib $\quad 5 \%$ grade 3 dizziness in both arms

No difference in late neurotoxicity

between arm A and B (not specified)

Arm A: grade $3 / 4$ somnolence $2.5 \%$

Arm $\mathrm{B}$ : $5 \%$, somnolence $2.5 \%$,

"no significant differences although headache and vomiting occurred more often in the WBRT arm"

None

None
Crade 3-5 toxicities arm A, B, C $11 \%, 41 \%$ and $49 \%$, respectively Arm C: fatigue, acne, diarrhe pneumonia, hyperkaliemia, muscle weakness

ILD $18 \%$, rash $9 \%$, fatigue $9 \%$

Rash, diarrhea, mucositis, oral trush (all 12.5\%), fatigue (37.5\%), hepatotoxicity (25\%),

hyponatriemia $(50 \%)$

(all grade 3) 15\% rash, $12.5 \%$

fatigue, $10 \%$ diarrhea, $2.5 \%$

nausea, $5 \%$ vomiting, $10 \%$

dehydration, $5 \%$ liver test

abnormalities, $2.5 \%$ pleural

effusion

Grade 3 toxicities arm A: 10\% anorexia, arm $B$ : none

No grade 4-5 toxicities

Grade 3/4 toxicities similar in Goth arms (70\%), except for rash (erlotinib 20\%, placebo 5\%) and fatigue (erlotinib $17.5 \%$, placebo $35 \%)$

QoL similar

Alopecia significantly more in WBRT + TKI arm compared to TKI alone ( $73 \%$ vs $4 \%$ )

Grade 3-4 toxicities arm A: mucositis $6.3 \%$, diarrhea $6.3 \%$ Arm B: lymphopenia $9.3 \%$, low CD4 2.3\%, liver test

abnormalities 9.3\%,fatigue $18.6 \%$ For arm A (gefitinib) not well defined: $70 \%$ rash, grade not mentioned

Arm B: grade 3-4 hematological

toxicities $41 \%$ fatigue $18.8 \%$, dyspnea $6.3 \%$

Median OS Arm A, B, C resp 13.4, 6.3 and $6.1 \mathrm{~m}(\mathrm{NS})$

7 patients FU imaging. 5/7 PR, 2/7 SD

7 patients evaluable, $75 \%$ DCR (25\% PR, 50\% SD)

ORR CNS 86\%

Overall median OS $11.8 \mathrm{~m}$

EGFRwt $9.3 \mathrm{~m}$

EGFR $+19.1 \mathrm{~m}$

ORR arm A and B resp 96 and $55 \%$

1 year OS arm A and B resp 35 vs

Neurological PFS both arms

$1.6 \mathrm{~m}$

Median OS arm A and B resp 3.4 and $2.9 \mathrm{~m}$

ORR of brain mets arm $A$ and $B$ resp $64 \%$ vs $27 \%$

DCR of brain mets $71 \%$ vs $42 \%$ Median PFS 10.6 vs $6.6 \mathrm{~m}$

Median OS 23.4 vs $14.8 \mathrm{~m}$

Arm A closed prematurely due to

futility

Median OS arm A and B resp 6.3

and $4.9 \mathrm{~m}$
RR arm A vs B $54 \%$ vs $47 \%$

Median OS arm A vs B 13.3 vs

$12.7 \mathrm{~m}$ 


\begin{tabular}{|c|c|c|c|c|c|c|}
\hline Trial, year & $\begin{array}{l}\text { Retrospective } \\
\text { (R) or pros- } \\
\text { pective }(\mathrm{P})\end{array}$ & $N$ & $\begin{array}{l}\text { Arms specified when } \\
\text { necessary }\end{array}$ & Neurological safety outcome & All other grade $3-5$ toxicities & Efficacy outcome \\
\hline $\begin{array}{l}\text { Ma et al. (2009) } \\
\text { [37] }\end{array}$ & $P$ & 21 & Single arm & $\begin{array}{l}14 \% \text { grade } 3 \text { headache, } 86 \% \text { grade } 3 \\
\text { alopecia } \\
\text { No grade } 4-5 \text { toxicities }\end{array}$ & $\begin{array}{l}\text { All domains of QoL improved } \\
\text { during treatment } \\
\text { Grade } 3 \text { toxicities: } 14.3 \% \\
\text { diarrhea, } 14 \% \text { nausea, } 14 \% \\
\text { vomiting, } 14 \% \text { fatigue } \\
\text { No grade } 4-5 \text { toxicities }\end{array}$ & $\begin{array}{l}\text { ORR } 81 \% \\
\text { Median PFS } 10.0 \mathrm{~m} \\
\text { Median OS } 13 \mathrm{~m}\end{array}$ \\
\hline \multicolumn{7}{|c|}{ Erlotinib or gefitinib and WBRT } \\
\hline $\begin{array}{l}\text { Cai et al. (2013) } \\
\text { [41] }\end{array}$ & $\mathrm{R}$ & 157 & $\begin{array}{l}\text { A: WBRT + TKI } \\
\text { B: WBRT only }\end{array}$ & $\begin{array}{l}\text { No significant neurotoxicity differences } \\
\text { between arm A and B. grade not } \\
\text { mentioned. }\end{array}$ & $\begin{array}{l}\text { Arm A: (grade not mentioned) } \\
\text { rash } 47.7 \% \text {, interstitial } \\
\text { pneumonia } 7.7 \% \text {, diarrhea } 7.7 \% \text {, }\end{array}$ & $\begin{array}{l}\text { Arm A RR 76.9\% DCR } 96.9 \% \\
\text { Arm B RR 70.7\% DCR } 89.1 \% \\
\text { Arm A PFS } 6.0 \mathrm{~m} \text {, OS } 10.6 \mathrm{~m} \\
\text { Arm B PFS } 3.4 \mathrm{~m} \text {, OS } 7.7 \mathrm{~m}\end{array}$ \\
\hline $\begin{array}{l}\text { Lee et al. (2012) } \\
\quad[43]\end{array}$ & $\mathrm{R}$ & 43 & $\begin{array}{l}\text { A: WBRT only } \\
\text { B: WBRT + TKI }\end{array}$ & None & $\begin{array}{l}\text { No } \geqslant \text { grade } 3 \text { toxicities in arm A. } \\
\text { In arm B: } 11 \% \text { grade } 3 \text { rash, } 5 \% \\
\text { grade } 3 \text { oral mucositis, } 5 \% \text { grade } \\
3 \text { otitis media }\end{array}$ & $\begin{array}{l}\text { RR EGFR + vs EGFRwt } 80 \% \text { vs } \\
46 \% \text {, EGFR + only predictor for } \\
\text { treatment response }\end{array}$ \\
\hline $\begin{array}{l}\text { Inamasu (conf } \\
\text { abstract) } \\
\quad(2012)[42]\end{array}$ & $\mathrm{R}$ & 18 & Single arm & None & No grade $\geqslant 3$ toxicities & $100 \%$ intracranial DCR \\
\hline \multicolumn{7}{|l|}{ Icotinib and WBRT } \\
\hline $\begin{array}{l}\text { Zhou et al. (conf } \\
\text { abstract) } \\
\text { (2014) [45] }\end{array}$ & $P$ & 15 & Single arm & $\begin{array}{l}\text { Compared to baseline no changes in } \\
\text { neurocognitive functioning at } 20 \text { weeks, } \\
\text { low dose vs high dose no differences }\end{array}$ & $\begin{array}{l}500 \mathrm{mg} \text { TID }=\text { DLT }(1 \text { pt grade } 3 \\
\text { ALAT increase, } 2 \text { pts grade } 3 \\
\text { nausea })\end{array}$ & $\begin{array}{l}\text { ORR } 80 \% \text {, DCR } 100 \% \text {, median PFS } \\
46 \text { weeks (intracranial } 78 \text { weeks) }\end{array}$ \\
\hline $\begin{array}{l}\text { Yun et al. (conf } \\
\text { abstract) } \\
\text { (2013) [44] }\end{array}$ & $P$ & 20 & Single arm & $\begin{array}{l}\text { No grade }>3 \text { toxicities, all grades: } \\
\text { headache } 35 \%\end{array}$ & $\begin{array}{l}\text { No grade }>3 \text { toxicities, all grades: } \\
\text { rash } 40 \% \text {, diarrhea } 15 \% \text {, nausea } \\
45 \% \text {, vomiting } 20 \% \text {, fatigue } 45 \%\end{array}$ & $\begin{array}{l}\text { ORR } 80 \% \\
\text { Median PFS EGFR + vs EGFRwt: } \\
\text { NR vs } 4.2 \text { months }\end{array}$ \\
\hline
\end{tabular}

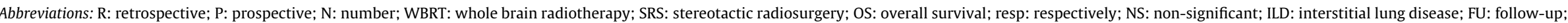

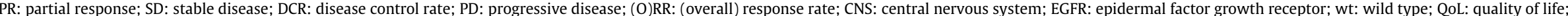
PFS: progression free survival; TKI: tyrosine kinase inhibitor; TID: three times a day; DLT: dose limiting toxicity; ALAT: alanine-aminotransferase; NR: not reached. 
Table 5

Ongoing or unpublished trials of TKI concurrent with cranial radiation.

\begin{tabular}{|c|c|c|c|c|c|c|c|c|}
\hline Trial, number & Trial type & $\begin{array}{l}\mathrm{N} \text { (to be } \\
\text { included) }\end{array}$ & EGFR mutation analysis & $\begin{array}{l}\text { WBRT/SRS } \\
\text { treatment }\end{array}$ & TKI treatment ( \pm comparator) & $\begin{array}{l}\text { Primary study } \\
\text { objective }\end{array}$ & Secondary study objectives & $\begin{array}{l}\text { Estimated primary } \\
\text { completion date }\end{array}$ \\
\hline \multicolumn{9}{|c|}{ Erlotinib and WBRT } \\
\hline NCT00871923 & $\begin{array}{l}\text { Phase II, single } \\
\text { center, open label, } \\
\text { single arm }\end{array}$ & 20 & Not mandatory & $\begin{array}{l}\text { WBRT } 35 \mathrm{~Gy}(14 \mathrm{f} / \\
2.5 \mathrm{~Gy})\end{array}$ & $\begin{array}{l}\text { Erlotinib } 150 \mathrm{mg} \text { once daily, start } \\
5 \text { days before WBRT, continue } \\
\text { during WBRT, continuation after } \\
\text { WBRT at investigators discretion }\end{array}$ & $\begin{array}{l}\text { Median OS } \\
\text { (designated as } \\
\text { safety issue) }\end{array}$ & Not provided & March 2016 \\
\hline NCT01887795 & $\begin{array}{l}\text { Phase III, } \\
\text { multicenter, open } \\
\text { label, randomized }\end{array}$ & 224 & $\begin{array}{l}\text { Mandatory, unclear in } \\
\text { description whether } \\
\text { only EGFR + patients } \\
\text { are included }\end{array}$ & $\begin{array}{l}\text { WBRT } 40 \text { Gy (20f/ } \\
2 \text { Gy) }\end{array}$ & $\begin{array}{l}\text { Arm A: Erlotinib } 150 \mathrm{mg} \text { once } \\
\text { daily, start } 5 \text { days before WBRT, } \\
\text { continue during WBRT } \\
\text { Afterwards erlotinib treatment } \\
\text { not specified } \\
\text { Arm B: WBRT only }\end{array}$ & $\begin{array}{l}\text { Time to } \\
\text { neurological } \\
\text { progression } \\
\text { (designated as } \\
\text { safety issue) }\end{array}$ & $\begin{array}{l}\text { OS } \\
\text { Response } \\
\text { QoL }\end{array}$ & August 2016 \\
\hline NCT01518621 & $\begin{array}{l}\text { Phase II, open } \\
\text { label, randomized }\end{array}$ & 150 & Not mandatory & $\begin{array}{l}\text { WBRT } 30 \text { Gy (10f/ } \\
3 \text { Gy) }\end{array}$ & $\begin{array}{l}\text { Arm A: Erlotinib } 150 \text { mg once } \\
\text { daily, start } 1 \text { day before WBRT, } \\
\text { continu during WBRT } \\
\text { Arm B: WBRT only }\end{array}$ & Median OS & $\begin{array}{l}\text { Safety, local control rate, } \\
\text { time to neurological } \\
\text { progression } \\
\text { (neuropsychological } \\
\text { testing), QoL, effect of } \\
\text { mutation status }\end{array}$ & Not provided \\
\hline NCT01130779 & $\begin{array}{l}\text { Phase II, open } \\
\text { label, single arm, } \\
\text { enrolling by } \\
\text { invitation }\end{array}$ & 23 & $\begin{array}{l}\text { Not mandatory, } \\
\text { however only patients } \\
\text { included on EGFR-TKI } \\
\text { treatment with good } \\
\text { extracranial control }\end{array}$ & $\begin{array}{l}\text { WBRT, SRS or } \\
\text { surgery } \\
\text { Dose radiotherapy } \\
\text { not specified }\end{array}$ & $\begin{array}{l}\text { Patients on EGFR-TKI treatment } \\
\text { and brain only PD: local } \\
\text { treatment with continuation of } \\
\text { EGFR-TKI }\end{array}$ & $\begin{array}{l}\text { PFS (not } \\
\text { designated as } \\
\text { safety issue) }\end{array}$ & $\begin{array}{l}\text { OS } \\
\text { Response rate } \\
\text { Time to treatment failure } \\
\text { Toxicity profiles }\end{array}$ & $\begin{array}{l}\text { August } 2010 \text { (study } \\
\text { information not updated) }\end{array}$ \\
\hline \multicolumn{9}{|c|}{ Gefitinib and WBRT } \\
\hline NCT01363557 & $\begin{array}{l}\text { Phase II, open } \\
\text { label, multicenter, } \\
\text { randomized }\end{array}$ & $\begin{array}{l}\text { Only } 1 \\
\text { enrolled }\end{array}$ & $\begin{array}{l}\text { Mandatory, only } \\
E G F R+\text { patients } \\
\text { included }\end{array}$ & $\begin{array}{l}\text { WBRT } 30 \text { Gy (10f/ } \\
3 \text { Gy) }\end{array}$ & $\begin{array}{l}\text { Arm A: gefitinib } 250 \mathrm{mg} \text { once } \\
\text { daily concurrent with WBRT } \\
\text { Arm B: gefitinib } 250 \mathrm{mg} \text { once } \\
\text { daily only }\end{array}$ & $\begin{array}{l}\text { Response rate } \\
\text { brain metastases } \\
\text { (designated as } \\
\text { safety issue) }\end{array}$ & $\begin{array}{l}\text { Neurological adverse events } \\
\text { PFS } \\
\text { OS }\end{array}$ & $\begin{array}{l}\text { Closed prematurely due to } \\
\text { poor accrual }\end{array}$ \\
\hline NCT02338011 & $\begin{array}{l}\text { Phase II/III, open } \\
\text { label, } 2 \text { arm, } \\
\text { randomized, } \\
\text { single center }\end{array}$ & 210 & $\begin{array}{l}\text { Mandatory, only } \\
E G F R+\text { patients } \\
\text { included }\end{array}$ & $\begin{array}{l}\text { WBRT } 30 \text { Gy (10f/ } \\
3 \text { Gy) }\end{array}$ & $\begin{array}{l}\text { Arm A: gefitinib } 250 \mathrm{mg} \text { once } \\
\text { daily concurrent with WBRT } \\
\text { Arm B: gefitinib } 250 \mathrm{mg} \text { once } \\
\text { daily only } \\
\text { Gefitinib in both arms until } \\
\text { progression }\end{array}$ & $\begin{array}{l}\text { PFS: intracranial, } \\
\text { extracranial and } \\
\text { overall } \\
\text { (designated as } \\
\text { safety issue) }\end{array}$ & $\begin{array}{l}\text { OS } \\
\text { Site of first progression } \\
\text { QoL } \\
\text { Mental status (MMSE) }\end{array}$ & November 2017 \\
\hline \multicolumn{9}{|c|}{ Icotinib and WBRT } \\
\hline NCT01926171 & $\begin{array}{l}\text { Phase IV, single } \\
\text { arm, open label }\end{array}$ & 80 & Not mandatory & $\begin{array}{l}\text { WBRT } 40 \text { Gy (20f/ } \\
2 \text { Gy) }\end{array}$ & $\begin{array}{l}\text { Icotinib (dose?) TID concurrent } \\
\text { with WBRT }\end{array}$ & $\begin{array}{l}\text { Response rate } \\
\text { brain metastases } \\
\text { (not designated as } \\
\text { safety issue) }\end{array}$ & $\begin{array}{l}\text { PFS } \\
\text { All cause progress/mortality } \\
\text { Safety }\end{array}$ & September 2014 \\
\hline
\end{tabular}


as this is seldom encountered with first generation EGFR-TKI [53] and was observed in $5-12.5 \%$ in the studies described in this review $[33,38,43]$. However, only one of these studies was randomized and in this trial, in both arms a systemic agent was given (temozolomide or gefitinib) [38]. Moreover, the technique of WBRT was very simple, thus including some mucosa in the irradiated volumes. Percentages of grade $\geqslant 3$ fatigue, nausea and vomiting do not seem to increase with EGFR-TKI concurrent with WBRT compared to monotherapy EGFR-TKI, although WBRT itself also can also cause fatigue, nausea and vomiting (usually $\leqslant$ grade 2 ) [55].

As safety was the focus of our review there was no restriction on the presence of a targetable mutation. As such, the question whether the potential increase in local CNS response rate and the prevention of tumor flare when the TKI is continued during cranial radiation outweighs the potential risk of clinically significant side effects is not answered. To date, there are no studies addressing this specific question in patients with an activating mutation. In two out of four studies that compare WBRT to concurrent EGFRTKI and WBRT, the response rate and OS were higher in patients that were treated with the combined treatment. However, in these trials EGFR mutation status was not tested or known only for a subset of patients. It was also unclear whether EGFR-mutated patients treated with WBRT only were afterwards treated with EGFR-TKI (i.e., possible undertreatment of these WBRT only patients) $[31,34,36,41]$.

As mentioned above, no studies were identified in which patients with an activating mutation/translocation developed brain metastases while on TKI treatment and were subsequently randomized to cranial radiotherapy with/without concurrent TKI. In current guidelines, no advice regarding TKI use during cranial radiotherapy is given $[2,3,22,23]$. In daily practice, the TKI is often discontinued for 4-5 times the half-life $\left(T^{1 / 2}\right)$ of the drug before start of cranial radiotherapy and is reinitiated a couple of days after cranial radiotherapy because of (neuro)toxicity concerns. For the first generation TKI's, $T^{1 / 2}$ is $36 \mathrm{~h}$ (erlotinib) to $40-42 \mathrm{~h}$ (gefitinibcrizotinib) [56-58]. T1/2 of icotinib is only six to eight hours [58]. As a result, TKI's are discontinued approximately two to three weeks because of cranial radiotherapy with the risk of a systemic disease flare-up. The 4-5 times the $T^{1 / 2}$ is based on the finding that after this time drugs are eliminated from the blood. However, it is unclear whether there are still remaining biological effects of the drug. Moreover, it is also unknown whether there is a dose-dependent effect for radiosensitisation. Furthermore, for other drugs like monoclonal antibodies $T 1 / 2$ is often more than one week (e.g., ipilimumab $T 1 / 215$ days) which makes it impossible due to the need for systemic disease control to discontinue these drugs for 4-5 times the $T 1 / 2$ [59]. As there is a lack of pre-clinical data for e.g., radiosensitisation and dose-dependency as well as remaining biological effects after discontinuation of the drug (TKI as well as monoclonal antibodies), this should be subjected to further research. Another question that is not evaluated in this review is what the best treatment sequence is for patients with an activating EGFR mutation or $A L K$-rearrangement with already a diagnosis of asymptomatic brain metastasis before commencement of a TKI. Based on available literature, patients with an activating EGFR mutation can start directly with an EGFR-TKI, as, despite the poor blood-brain-barrier penetration of first generation EGFR-TKIs, the cerebral response rate is more than $80 \%$ [7]. For $A L K$-rearranged patients, data are less clear. In the retrospective pooled analysis of the subgroup of patients with previously untreated brain metastases included in the PROFILE 1005 and 1007 studies, cerebral response and disease control rate were only $18 \%$ and $53 \%$, respectively, and the CNS was the initial site of progression in $70 \%$ of patients [8]. One can argue that in this patient population, cranial radiotherapy can be considered first.
Also, the place of the second and third generation TKI's (EGFR: afatinib, AZD9291, CO-1686, ALK: ceritinib, alectinib) should be determined as these agents have a better penetration in the CSF compared to first generation TKI's and cranial responses are found with these agents in patients who develop brain metastases when they have already been treated with first generation TKI [15,6062]. Another option that could be explored in this patient population is the use of SRS without WBRT, even for multiple (five to ten) brain metastases. This because recently it was found that results for SRS alone did not differ between patients with two to four brain metastases compared to five to ten metastases [63]. The advantages for SRS/SRT without WBRT are that both cognition and quality of life are superior with SRS/SRT alone [64]. Moreover, local control rates are durable (12-month local control rate between $50 \%$ and more than $80 \%$ dependent on radiation dose and volume of brain metastases) and complication rate is low (grade $\geqslant 3$ adverse events less than $5 \%$ ) $[63,65,66]$. However, because of number and/or volume of brain metastases, not all patients are suitable for SRS/SRT and WBRT still is an option in this patient population. Although there are some studies investigating WBRT concurrently with an EGFR-TKI (overview in Table 5), there are currently no ongoing studies in patients with an activating EGFR-mutation oligoprogressive in the brain while on EGFR-TKI treatment.

In summary, although there are arguments that EGFR-TKI can be safely applied concurrent with WBRT, there is no high-level evidence to support this. With the addition of SRS/SRT to WBRT, severe (grade 3-5) toxicities may increase, although further studies are needed [34]. For $A L K$-rearranged patients no data are available. This review stresses the need for high quality studies evaluating the use of TKI with concurrent radiotherapy in patients with an activating EGFR-mutation and/or $A L K$-rearrangement as well as for the further evaluation of the place of second and third generation TKI's and SRS/SRT in this selected patient population.

\section{Conflict of interest}

No conflict of interest.

\section{Funding}

none.

\section{Contributions}

L. Hendriks: conception and design of study, acquisition of data, analysis and interpretation of data, drafting the article, final approval of article.

J. Schoenmaekers: acquisition of data, analysis and interpretation of data, critically revising the article, final approval of article.

J. Zindler: analysis and interpretation of data, critically revising the article, final approval of article.

D. Eekers: analysis and interpretation of data, critically revising the article, final approval of article.

A. Hoeben: analysis and interpretation of data, critically revising the article, final approval of article.

D. De Ruysscher: analysis and interpretation of data, drafting and critically revising the article, final approval of article.

A.-M. Dingemans: Conception and design of study, analysis and interpretation of data, drafting and critically revising the article, final approval of article.

\section{Appendix A. Supplementary data}

Supplementary data associated with this article can be found, in the online version, at http://dx.doi.org/10.1016/j.ctrv.2015.05.005. 


\section{References}

[1] Stella GM, Luisetti M, Pozzi E, Comoglio PM. Oncogenes in non-small-cell lung cancer: emerging connections and novel therapeutic dynamics. Lancet Respir Med 2013;1:251-61.

[2] NCCN Guidelines version 1.2015 Non-Small Cell Lung Cancer. 2014.

[3] Reck M, Popat S, Reinmuth N, et al. Metastatic non-small-cell lung cancer (NSCLC): ESMO Clinical Practice Guidelines for diagnosis, treatment and follow-up. Ann Oncol 2014;25(Suppl. 3):27-39.

[4] Doebele RC, Lu X, Sumey C, et al. Oncogene status predicts patterns of metastatic spread in treatment-naive nonsmall cell lung cancer. Cancer 2012;118:4502-11.

[5] Shaw AT, Kim DW, Nakagawa K, et al. Crizotinib versus chemotherapy in advanced ALK-positive lung cancer. N Engl J Med 2013;368:2385-94.

[6] Rangachari D, Yamaguchi N, VanderLaan PA, et al. Brain metastases in patients with EGFR-mutated or ALK-rearranged non-small-cell lung cancers. Lung cancer 2015.

[7] Zimmermann S, Dziadziuszko R, Peters S. Indications and limitations of chemotherapy and targeted agents in non-small cell lung cancer brain metastases. Cancer Treat Rev 2014:40:716-22.

[8] Costa DB, Shaw AT, Ou SH, et al. Clinical experience with crizotinib in patients with advanced ALK-rearranged non-small-cell lung cancer and brain metastases. J Clin Oncol 2015.

[9] Heon S, Yeap BY, Britt GJ, et al. Development of central nervous system metastases in patients with advanced non-small cell lung cancer and somatic EGFR mutations treated with gefitinib or erlotinib. Clin Cancer Res 2010;16:5873-82.

[10] Heon S, Yeap BY, Lindeman NI, et al. The impact of initial Gefitinib or Erlotinib versus chemotherapy on central nervous system progression in advanced nonsmall cell lung cancer with EGFR mutations. Clin Cancer Res 2012;18:4406-14.

[11] Patel S, Rimner A, Foster A, et al. Risk of brain metastasis in EGFR-mutant NSCLC treated with erlotinib: a role for prophylactic cranial irradiation? Int J Radiat Oncol Biol Phys 2014;90:S643-4.

[12] Weickhardt AJ, Scheier B, Burke JM, et al. Local ablative therapy of oligoprogressive disease prolongs disease control by tyrosine kinase inhibitors in oncogene-addicted non-small-cell lung cancer. J Thorac Oncol 2012;7:1807-14.

[13] Park SJ, Kim HT, Lee DH, et al. Efficacy of epidermal growth factor receptor tyrosine kinase inhibitors for brain metastasis in non-small cell lung cancer patients harboring either exon 19 or 21 mutation. Lung cancer 2012;77:556-60.

[14] Nishie K, Kawaguchi T, Tamiya A, et al. Epidermal growth factor receptor tyrosine kinase inhibitors beyond progressive disease: a retrospective analysis for Japanese patients with activating EGFR mutations. J Thorac Oncol 2012;7:1722-7.

[15] Hoffknecht P, Tufman A, Wehler T, et al. Efficacy of the irreversible ErbB family blocker afatinib in epidermal growth factor receptor (EGFR) tyrosine kinase inhibitor (TKI)-pretreated non-small-cell lung cancer patients with brain metastases or leptomeningeal disease. J Thorac Oncol 2015;10:156-63.

[16] Ou SH, Janne PA, Bartlett CH, et al. Clinical benefit of continuing ALK inhibition with crizotinib beyond initial disease progression in patients with advanced ALK-positive NSCLC. Ann Oncol 2014;25:415-22.

[17] Chinnaiyan P, Huang S, Vallabhaneni G, et al. Mechanisms of enhanced radiation response following epidermal growth factor receptor signaling inhibition by erlotinib (Tarceva). Cancer Res 2005;65:3328-35.

[18] Zhuang HQ Sun J, Yuan ZY, et al. Radiosensitizing effects of gefitinib at different administration times in vitro. Cancer Sci 2009;100:1520-5.

[19] Bokobza SM, Jiang Y, Weber AM, et al. Short-course treatment with gefitinib enhances curative potential of radiation therapy in a mouse model of human non-small cell lung cancer. Int J Radiat Oncol Biol Phys 2014;88:947-54.

[20] Dai Y, Wei Q, Schwager C, et al. Synergistic effects of crizotinib and radiotherapy in experimental EML4-ALK fusion positive lung cancer. Radiother Oncol 2015;114:173-81.

[21] Das AK, Sato M, Story MD, et al. Non-small-cell lung cancers with kinase domain mutations in the epidermal growth factor receptor are sensitive to ionizing radiation. Cancer Res 2006;66:9601-8.

[22] NCCN guidelines Version 2.2014 Central Nervous System Cancers. 2014.

[23] Tsao MN, Rades D, Wirth A, et al. Radiotherapeutic and surgical management for newly diagnosed brain metastasis(es): An American Society for Radiation Oncology evidence-based guideline. Pract Radiat Oncol 2012;2:210-25.

[24] Lilenbaum R, Samuels M, Wang X, et al. A phase II study of induction chemotherapy followed by thoracic radiotherapy and erlotinib in poor-risk stage III non-small-cell lung cancer: results of CALGB 30605 (Alliance)/RTOG 0972 (NRG). J Thorac Oncol 2015;10:143-7.

[25] Xu Y, Zhang Y, Ma S. EGFR inhibitors with concurrent thoracic radiation therapy for locally advanced non-small cell lung cancer. Lung cancer 2011;73:249-55

[26] Koh PK, Faivre-Finn C, Blackhall FH, De Ruysscher D. Targeted agents in nonsmall cell lung cancer (NSCLC): clinical developments and rationale for the combination with thoracic radiotherapy. Cancer Treat Rev 2012;38:626-40.

[27] Ramella S, Alberti AM, Cammilluzzi E, et al. Erlotinib and concurrent chemoradiation in pretreated NSCLC patients: radiobiological basis and clinical results. Biomed Res Int 2013;2013:403869.

[28] Chaft JE, Oxnard GR, Sima CS, et al. Disease flare after tyrosine kinase inhibitor discontinuation in patients with EGFR-mutant lung cancer and acquired resistance to erlotinib or gefitinib: implications for clinical trial design. Clin Cancer Res 2011;17:6298-303.

[29] Pop O, Pirvu A, Toffart AC, Moro-Sibilot D. Disease flare after treatment discontinuation in a patient with EML4-ALK lung cancer and acquired resistance to crizotinib. J Thorac Oncol 2012;7:e1-2.

[30] da Costa Santos CM CM, de Mattos Pimenta CA, Nobre MR. The PICO strategy for the research question construction and evidence search. Rev Lat Am Enfermagem 2007:15:508-11.

[31] Lee SM, Lewanski CR, Counsell N, et al. Randomized trial of erlotinib plus whole-brain radiotherapy for NSCLC patients with multiple brain metastases. J Natl Cancer Inst 2014:106.

[32] Lind JS, Lagerwaard FJ, Smit EF, Senan S. Phase I study of concurrent whole brain radiotherapy and erlotinib for multiple brain metastases from nonsmall-cell lung cancer. Int J Radiat Oncol Biol Phys 2009;74:1391-6.

[33] Olmez I, Donahue BR, Butler JS, et al. Clinical outcomes in extracranial tumor sites and unusual toxicities with concurrent whole brain radiation (WBRT) and Erlotinib treatment in patients with non-small cell lung cancer (NSCLC) with brain metastasis. Lung Cancer 2010;70:174-9.

[34] Sperduto PW, Wang M, Robins HI, et al. A phase 3 trial of whole brain radiation therapy and stereotactic radiosurgery alone versus WBRT and SRS with temozolomide or erlotinib for non-small cell lung cancer and 1 to 3 brain metastases: Radiation Therapy Oncology Group 0320. Int J Radiat Oncol Biol Phys 2013;85:1312-8.

[35] Welsh JW, Komaki R, Amini A, et al. Phase II trial of erlotinib plus concurrent whole-brain radiation therapy for patients with brain metastases from nonsmall-cell lung cancer. J Clin Oncol 2013:31:895-902.

[36] Zhuang H, Yuan Z, Wang J, et al. Phase II study of whole brain radiotherapy with or without erlotinib in patients with multiple brain metastases from lung adenocarcinoma. Drug Des Devel Ther 2013;7:1179-86.

[37] Ma S, Xu Y, Deng Q, Yu X. Treatment of brain metastasis from non-small cell lung cancer with whole brain radiotherapy and Gefitinib in a Chinese population. Lung cancer 2009;65:198-203.

[38] Pesce GA, Klingbiel D, Ribi K, et al. Outcome, quality of life and cognitive function of patients with brain metastases from non-small cell lung cancer treated with whole brain radiotherapy combined with gefitinib or temozolomide. A randomised phase II trial of the Swiss Group for Clinical Cancer Research (SAKK 70/03). Eur J Cancer 2012;48:377-84.

[39] Wang F, Ning F, Liu C, et al. Comparison of Gefitinib versus VMP in the combination with radiotherapy for multiple brain metastases from non-small cell lung cancer. Cell Biochem Biophys 2014.

[40] Zeng YD, Zhang L, Liao H, et al. Gefitinib alone or with concomitant whole brain radiotherapy for patients with brain metastasis from non-small-cell lung cancer: a retrospective study. Asian Pac J Cancer Prev 2012;13:909-14.

[41] Cai Y, Wang JY, Liu H. Clinical observation of whole brain radiotherapy concomitant with targeted therapy for brain metastasis in non-small cell lung cancer patients with chemotherapy failure. Asian Pac J Cancer Prev 2013;14:5699-703.

[42] Inamasu E, Toyokawa G, Shiraishi Y, et al. A feasibility of concurrent whole brain radiation plus epidermal growth factor receptor-tyrosine kinase inhibitor for non-small cell lung cancer. J Thorac Oncol 2012;7:S479.

[43] Lee HL, Chung TS, Ting LL, et al. EGFR mutations are associated with favorable intracranial response and progression-free survival following brain irradiation in non-small cell lung cancer patients with brain metastases. Radiat Oncol 2012;7:181.

[44] Yun F, Huang ZY, Gong L, et al. Icotinib and whole brain radiotherapy (WBRT) for patients with brain metastases from non-small cell lung cancer (NSCLC): Preliminary results of a phase II study. J Clin Oncol 2013:31.

[45] Zhou L, He J, Xiong W, et al. Phase I trial of icotinib combined with whole brain radiotherapy for EGFR-mutated non-small cell lung cancer patients with brain metastases. J Clin Oncol 2014;32(Suppl. 1).

[46] Costa DB, Shaw AT, Ou SHI, et al. Clinical experience with crizotinib in patients with advanced ALK-rearranged nonsmall cell lung cancer and brain metastases in profile 1005 and profile 1007. J Thorac Oncol 2013;8:S294-5.

[47] Costa DB, Kobayashi S, Pandya SS, et al. CSF concentration of the anaplastic lymphoma kinase inhibitor crizotinib. J Clin Oncol 2011;29:e443-5.

[48] Deng Y, Feng W, Wu J, et al. The concentration of erlotinib in the cerebrospinal fluid of patients with brain metastasis from non-small-cell lung cancer. Mol Clin Oncol 2014;2:116-20.

[49] Togashi Y, Masago K, Masuda S, et al. Cerebrospinal fluid concentration of gefitinib and erlotinib in patients with non-small cell lung cancer. Cancer Chemother Pharmacol 2012;70:399-405.

[50] Andrews DW, Scott CB, Sperduto PW, et al. Whole brain radiation therapy with or without stereotactic radiosurgery boost for patients with one to three brain metastases: phase III results of the RTOG 9508 randomised trial. Lancet 2004;363:1665-72.

[51] Shi L, Tang J, Tong L, Liu Z. Risk of interstitial lung disease with gefitinib and erlotinib in advanced non-small cell lung cancer: a systematic review and meta-analysis of clinical trials. Lung Cancer 2014;83:231-9.

[52] Melosky B, Hirsh V. Management of common toxicities in metastatic NSCLC related to anti-lung cancer therapies with EGFR-TKIs. Front Oncol 2014;4:238.

[53] Reck M, Mok T, Wolf J, et al. Reviewing the safety of erlotinib in non-small cell lung cancer. Exp Opin Drug Saf 2011:10:147-57.

[54] Yoshida T, Yamada K, Azuma K, et al. Comparison of adverse events and efficacy between gefitinib and erlotinib in patients with non-small-cell lung cancer: a retrospective analysis. Med Oncol 2013;30:349. 
[55] Cao KI, Lebas N, Gerber S, et al. Phase II randomized study of whole-brain radiation therapy with or without concurrent temozolomide for brain metastases from breast cancer. Ann Oncol 2015;26:89-94.

[56] Lu JF, Eppler SM, Wolf J, et al. Clinical pharmacokinetics of erlotinib in patients with solid tumors and exposure-safety relationship in patients with non-small cell lung cancer. Clin Pharmacol Ther 2006;80:136-45.

[57] Ranson M, Hammond LA, Ferry D, et al. ZD1839, a selective oral epiderma growth factor receptor-tyrosine kinase inhibitor, is well tolerated and active in patients with solid, malignant tumors: results of a phase I trial. J Clin Oncol 2002;20:2240-50

[58] Li C, Alvey C, Bello A, et al. Pharmacokinetics (PK) of crizotinib (PF-02341066) in patients with advanced non-small cell lung cancer (NSCLC) and other solid tumors. J Clin Oncol 2011;29(Suppl.; abstr e13065).

[59] Feng Y, Masson E, Dai D, et al. Model-based clinical pharmacology profiling of ipilimumab in patients with advanced melanoma. $\mathrm{Br} J$ Clin Pharmaco 2014;78:106-17.

[60] Kim DW, Mehra R, Tan D, et al. Ceritinib in advanced anaplastic lymphoma kinase (ALK)-rearranged (ALK+) non-small cell lung cancer (NSCLC) Results of the ASCEND-1 trial. J Clin Oncol 2014;32(Suppl.; abstr 8003).
[61] Sequist L, Soria J-C, Gadgeel S, et al. First-in-human evaluation of CO-1686, an irreversible, highly selective tyrosine kinase inhibitor of mutations of EGFR (activating and T790M). J Clin Oncol 2014;32(Suppl.; abstr 8010).

[62] Nakagawa K, Hida T, Seto T, et al. Antitumor activity of alectinib (CH5424802 R05424802) for ALK-rearranged NSCLC with or without prior crizotinib treatment in bioequivalence study. J Clin Oncol 2014;32(Suppl.; abstr 8103).

[63] Yamamoto M, Serizawa T, Shuto T, et al. Stereotactic radiosurgery for patients with multiple brain metastases (JLGK0901): a multi-institutional prospective observational study. Lancet Oncol 2014;15:387-95.

[64] Chang EL, Wefel JS, Hess KR, et al. Neurocognition in patients with brain metastases treated with radiosurgery or radiosurgery plus whole-brain irradiation: a randomised controlled trial. Lancet Oncol 2009;10:1037-44.

[65] Rodrigues G, Zindler J, Warner A, Lagerwaard F. Recursive partitioning analysis for the prediction of stereotactic radiosurgery brain metastases lesion control. Oncologist 2013;18:330-5.

[66] Wiggenraad R, Verbeek-de Kanter A, Kal HB, et al. Dose-effect relation in stereotactic radiotherapy for brain metastases. A systematic review. Radiother Oncol 2011;98:292-7. 